Cite this: RSC Adv., 2013, 3, 20016

Received 7th June 2013

Accepted 12th August 2013

DOI: $10.1039 /$ c3ra42840k

www.rsc.org/advances

\section{Capsule-free fluid delivery and beam-induced electrodeposition in a scanning electron microscope $\uparrow$}

\author{
Steven J. Randolph, ${ }^{* a}$ Aurelien Botman ${ }^{\mathrm{a}}$ and Milos Toth $^{\mathrm{b}}$ \\ Gold coated borosilicate nanocapillaries are used to locally deliver aqueous, electrolytic $\mathrm{CuSO}_{4}$ solution into \\ the low vacuum chamber of an environmental scanning electron microscope (ESEM). Capillary flow of the \\ liquid is induced by bringing a nanocapillary into contact with a substrate. A microscopic droplet is \\ stabilized by controlling the droplet evaporation rate with the substrate temperature and the pressure \\ of $\mathrm{H}_{2} \mathrm{O}$ vapor injected into the vacuum chamber. An electron beam is admitted to the droplet through a \\ pressure limiting aperture. Electrochemical reduction of aqueous $\mathrm{Cu}^{2+}$ to solid, high purity, deposited $\mathrm{Cu}$ \\ is achieved by biasing the nanocapillary and supplying current by the beam which acts as a virtual \\ cathode and enables electrodeposition on both conductive and insulating substrates. Delivery of liquids \\ into vacuum enables localized, capsule-free beam induced electrochemistry, opening new pathways for \\ direct-write nano and micro-lithography via beam induced electrodeposition.
}

\section{Introduction}

The ability to deliver liquids into vacuum has enabled electron microscopy ${ }^{\mathbf{1}, 2}$ of liquid-solid interactions at the micro- and nano-scales. ${ }^{3-12}$ Liquid delivery is typically achieved by one of three methods. In the first, used in environmental electron microscopy, a differentially pumped electron column is separated from the specimen chamber by pressure limiting apertures. A liquid is stabilized on a cooled solid substrate by controlling the substrate temperature and the partial pressure of the vapor inside the vacuum chamber. The main shortcoming of this approach is that it does not enable localized, controlled delivery of a liquid to a specific point at the substrate surface. The second method entails the containment of a liquid in a capsule, and admittance of an electron beam through a thin, electron transparent membrane. This approach is advantageous in that it provides control over liquid flow through the capsule, and studies of chemical reactions at the liquidmembrane interface. The main limitations of this approach stem from the rigid geometric constraints of the solid-liquid system. The entire capsule must either be sufficiently thin for transmission electron microscopy, or can only be analyzed by the emissive backscattered, cathodoluminescence or X-ray signals encountered in electron microscopy. Furthermore, the method is poorly suited to studies of wetting and spreading at solid-vapor interfaces. Finally, and most relevant to the work

${ }^{a}$ FEI Company, 5350 NE Dawson Creek Drive, Hillsboro, OR 97214-5793, USA. E-mail: steven.randolph@fei.com

${ }^{b}$ School of Physics and Advanced Materials, University of Technology, Sydney, P.O. Box 123, Broadway, New South Wales 2007, Australia

$\dagger$ Electronic supplementary information (ESI) available. See DOI: $10.1039 / \mathrm{c} 3 \mathrm{ra} 42840 \mathrm{k}$ presented here, delivery of liquids through micro- or nanocapillaries into vacuum has previously been accomplished by means of an additional gas source that accelerates and confines liquid jets directed into microscopes for purposes of ESEM imaging ${ }^{\mathbf{1 3}}$ and electron crystallography. ${ }^{\mathbf{1 4}}$

Here we describe a capsule-free, nanocapillary method for localized delivery of liquids onto a solid substrate inside the specimen chamber of a scanning electron microscope (SEM). The method employs capillary (rather than hydrostatic) flow, thereby enabling the formation of stable, microscopic droplets at specific locations of a solid-vacuum interface. We use the method to deliver aqueous $\mathrm{CuSO}_{4}$, which is then used as a precursor for localized electrodeposition of $\mathrm{Cu}$ induced by the electron beam. The results demonstrate how a capillary-flowbased microfluidic system can be interfaced to the vacuum chamber of an ESEM, for purposes of manipulating a reactive liquid with an electron beam.

\subsection{Direct-write electron beam induced deposition}

Gas-mediated electron beam induced deposition (EBID) ${ }^{\mathbf{1 5 - 1 8}}$ is a direct-write nanofabrication process in which gaseous precursor molecules are injected into an electron microscope, adsorb to a solid substrate, and are dissociated by energetic electrons. The molecules contain a desirable material, such as a metal center, that remains on the surface in the form of a deposit. Ideally, any remaining reaction byproducts are volatile, desorb spontaneously and do not alter the nominal composition of the deposit. However, in practice, a primary drawback to the EBID technique is impurity incorporation caused by ligand dissociation, incomplete precursor dissociation, and low volatility of ligand fragments. ${ }^{\mathbf{1 9 - 2 1}}$ This is particularly problematic in the case of organometallic precursors, which are often used for 
EBID. Deposits grown from organometallics typically consist of metal nanocrystallites embedded in an amorphous carbon matrix. A large fraction of the EBID literature is focused on minimizing carbon incorporation ${ }^{15-18,21-26}$ by optimizing precursor molecule or gas mixture composition, growth conditions, or by using post-growth processing treatments.

Chemicals that can be used as precursors in EBID are limited by the need to work in vacuum, stability, and the properties of the surface-adsorbed precursor molecules and fragments generated during growth. The range can, in principle, be extended significantly by the use of chemicals that are in the liquid rather than gas state during EBID. For example, an enormous range of aqueous ionic solutions are routinely used for chemical electrodeposition, a common and well studied process for the growth of highly pure metals. Recent papers report liquid phase EBID (LP-EBID) of Pt, Au and Si performed using a cell that encapsulates the precursor and isolates it from the electron microscope vacuum chamber..$^{3-7}$ LP-EBID is therefore limited to the growth of small structures onto the underside of thin (electron transparent) substrates. The capillary flow liquid delivery method described in the present paper eliminates this limitation and enables an alternate approach to LPEBID using borosilicate nanocapillaries (NCs) which are used to deliver aqueous, electrolytic solutions to bulk substrates. The solution is stabilized thermodynamically using environmental SEM (ESEM) $)^{27-29}$ which enables stable SEM operation near 100\% relative humidity $(\mathrm{RH})$. In this manner, an electrolyte is delivered to a substrate, the NC can be biased, and the electron beam can function as a virtual cathode that facilitates localized electrochemical reduction and deposition.

\section{Materials and methods}

The microscope used in all experiments was an FEI Company Quanta 3D FEG DualBeam ${ }^{\mathrm{TM}}$, which includes a field emission source ESEM and a gallium liquid metal ion source focused ion beam (FIB). ${ }^{30}$ The system was fitted with a temperaturecontrolled Peltier heating/cooling stage that allowed for substrate temperatures in the range of $-10{ }^{\circ} \mathrm{C}$ to $60{ }^{\circ} \mathrm{C}$. Substrate cooling in conjunction with low vacuum operation allowed for operation at or below $100 \% \mathrm{RH}$. For instance, at $667 \mathrm{~Pa}$ of water vapor, and a substrate temperature of $0{ }^{\circ} \mathrm{C}$, liquid water is in equilibrium with water vapor, and these conditions are within the operating range of a standard ESEM. Further details on imaging of wet and liquid samples can be found elsewhere. ${ }^{12,28}$

Standard cleaning procedures were used after cleaving wafer segments to remove trace hydrocarbon contaminants and other unwanted particles. Typically, samples are cleaved and treated in ultrasonic baths in subsequent steps of 15 minutes each of acetone, isopropanol and deionized water. Samples are then baked at $150{ }^{\circ} \mathrm{C}$ and allowed to cool in air. Samples not used immediately are typically stored in a $50{ }^{\circ} \mathrm{C}$ incubating oven.

Aqueous copper sulfate, $\mathrm{CuSO}_{4}$, was used as the precursor for electrolytic deposition. To prepare solutions, $\mathrm{CuSO}_{4} \cdot 5 \mathrm{H}_{2} \mathrm{O}$ (Sigma-Aldrich part number 203165) was dissolved in ultrapure, trace analysis grade water (Sigma-Aldrich part number 14211).
The solution used in experiments had to be diluted to at least $0.8 \mathrm{mM}$ due to continual evaporation of water from the solution (below 100\% RH). Higher concentrations can result in salt crystal formation and blockage of the NC.

The liquid precursor was delivered to a substrate using a customized liquid injector based on a standard capillary-style Gas Injection System (GIS) ${ }^{15}$ typically employed in DualBeam ${ }^{\mathrm{TM}}$ systems. An assembly was designed to attach a glass capillary and enable positioning above a substrate in a manner analogous to a standard GIS. The NCs used were borosilicate glass capillaries formed using standard pulling techniques. This allowed inner diameters as small as $100 \mathrm{~nm}$ to be obtained, which ensured negligible hydrostatic-pressure-driven flow into the vacuum chamber (relative to capillary flow induced by bringing an NC into contact with a substrate). To enable electrical biasing of an NC, a gold coating of approximately $50 \mathrm{~nm}$ was sputtered onto the outer surface, and the NC tip was milled with the FIB to achieve the desired inner diameter.

NCs were filled with $\mathrm{CuSO}_{4}$ electrodeposition precursor using a micropipette and microloader system (Eppendorf part number 5242 956.003). Several microliters were injected into the backside of an NC (bore diameter $\sim 0.5 \mathrm{~mm}$ ) which was then

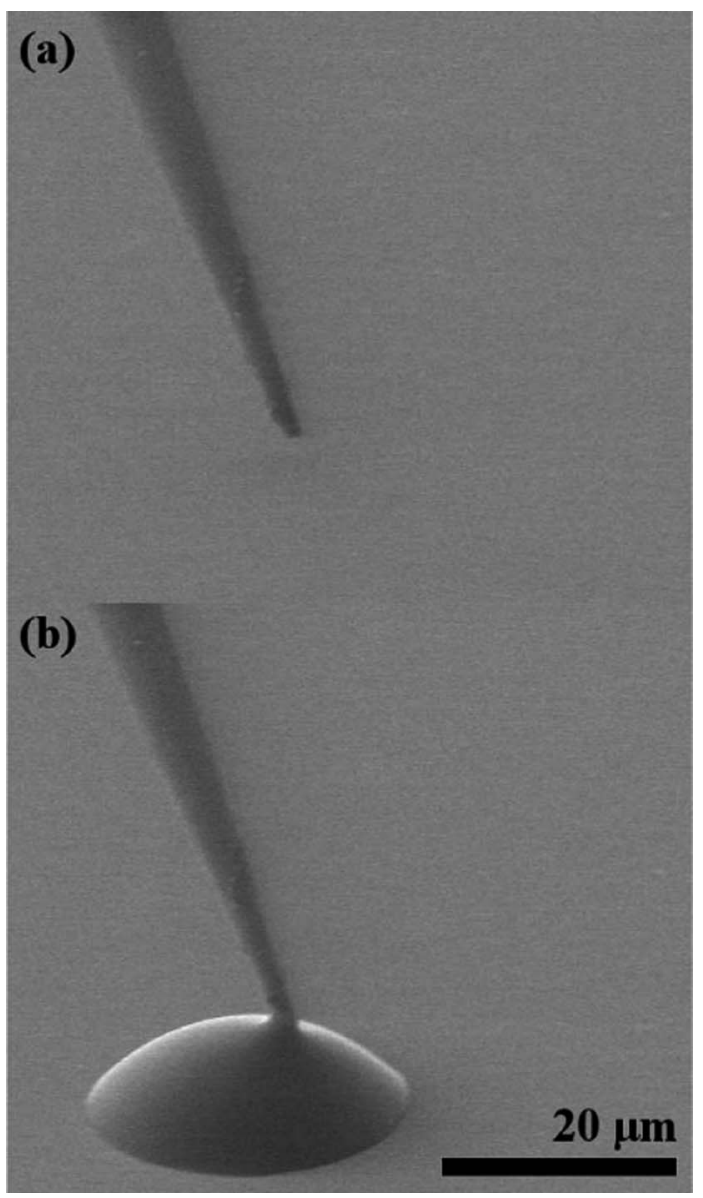

Fig. 1 ESEM electron micrograph of NC touchdown used to initiate capillary flow of electrolyte onto the sample surface. Prior to touchdown (a) no liquid is flowing. Upon contact with the surface, flow of electrolyte initiates and a stable droplet forms on the substrate $(b)$ [sample tilt $=45^{\circ}$ ]. 
sealed to prevent leakage during pump-down. The NC was then mounted to the GIS assembly inside the vacuum chamber and electrically connected to enable biasing up to $30 \mathrm{~V}$ with respect to the chamber ground. The biased NC acted as the anodic surface in all electrochemistry experiments. For additional details on NC mounting and vacuum sealing, ESI $\uparrow$ has been provided.

The ESEM was pumped to approximately $600 \mathrm{~Pa}$ of $\mathrm{H}_{2} \mathrm{O}$. The substrate, mounted on the Peltier stage, was then cooled to approximately $-0.5{ }^{\circ} \mathrm{C}$, resulting in an environment of just below $100 \% \mathrm{RH}$. It is important to remain below $100 \%$ to prevent rapid, delocalized condensation of water on the substrate. The NC was aligned within the electron beam field of view (Fig. 1a), and the substrate was raised to make contact with the NC and initiate capillary flow of the electrolyte (Fig. 1b). The pressure and temperature were used to modulated the flow rate of the liquid. The liquid droplet seen in Fig. $1 \mathrm{~b}$ can remain stable for an extended period of time (high $\mathrm{RH}$ ), or evaporate rapidly (low $\mathrm{RH}$ ) if the NC is retracted from the droplet. In all experiments discussed here, the NC remained inserted in the droplet until each experiment was completed. In cases where the NC had to be electrically isolated from the substrate, flow was first initiated by contacting the NC to the sample. After flow was established, the sample was lowered a few microns until the NC was only in contact with the droplet.

Experiments requiring area patterning with the electron beam were performed in a reduced area imaging mode as opposed to the built in patterning engine. The primary reason for this choice was to enable high quality real time monitoring of the process in ESEM mode. This required single pixel dwell times ranging between 1 and $10 \mu \mathrm{s}$.

\section{Results}

From hereon, the term 'virtual cathode' is used to refer to an electron beam acting as a current source that drives an electrodeposition process at a solid-electrolyte interface. Our results are focused on two distinct deposition configurations. In the first configuration, termed the beam-on-insulator virtual cathode (BIVC), the liquid is delivered onto an electrically insulating surface with concurrent electron beam irradiation of the insulator. The second configuration, beam-on-conductor virtual cathode (BCVC), is the case in which the electrolyte is delivered onto a conductive substrate with concurrent electron beam irradiation of the conductive feature. BCVC was performed on pre-fabricated metal electrodes, and on Pt features that had been pre-patterned in situ by ion beam induced deposition (IBID). ${ }^{15}$

The sample used for BIVC was a $200 \mathrm{~nm}$ film of thermal $\mathrm{SiO}_{2}$ on a $\mathrm{Si}$ substrate. It was pre-patterned with fiduciary marks to enable location of the experimental areas after processing. The outline of a $400 \mu \mathrm{m}$ by $35 \mu \mathrm{m}$ rectangle was milled through the $\mathrm{SiO}_{2}$ and into the $\mathrm{Si}$ using a $1 \mathrm{nA}$ FIB. In the center of the rectangle, several shallow lines were milled into the $\mathrm{SiO}_{2}$ (but not fully back to the Si) as finer fiduciary marks (Fig. 2).

Following pre-pattering of the sample in high vacuum, the system was switched to ESEM mode, $\mathrm{CuSO}_{4}$ was delivered to the

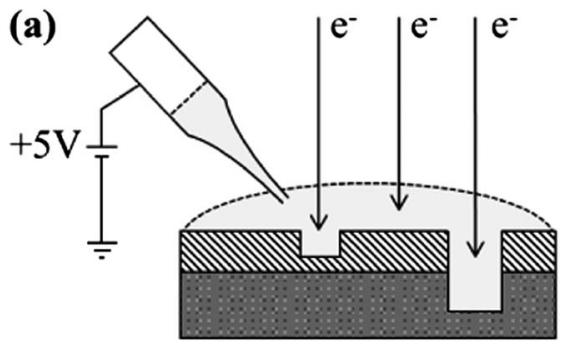

(b)

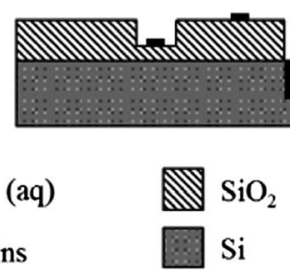

Fig. 2 Schematic illustration of the BIVC configuration used for electron beam induced electrodeposition of $\mathrm{Cu}$. The $\mathrm{NC}$ was used to deliver $\mathrm{CuSO}_{4}$ liquid to a region of $\mathrm{SiO}_{2}$ which contained trenches that had been milled into and through the $\mathrm{SiO}_{2}$ film, into a Si substrate (a). Electron beam irradiation resulted in trace quantities of $\mathrm{Cu}$ deposition on $\mathrm{SiO}_{2}$, and large quantities of $\mathrm{Cu}$ on the exposed $\mathrm{Si}$ surface (b).

$\mathrm{SiO}_{2}$ surface and the liquid was stabilized as discussed previously. The $\mathrm{CuSO}_{4}$ droplet overlapped both the lines milled back to the $\mathrm{Si}$ and the lines that terminated in the $\mathrm{SiO}_{2}$, as shown schematically in Fig. 2a. Two experiments were performed using this configuration. First, the $\mathrm{NC}$ was biased to $+5 \mathrm{~V}$ and the electron beam was scanned over an area of approximately $350 \mu \mathrm{m}^{2}$ that extended over both regions shown in Fig. 2a (i.e., electrolyte-on- $\mathrm{SiO}_{2}$ and electrolyte-on-Si). The electron beam energy, current and exposure time were $30 \mathrm{keV}, 16 \mathrm{nA}$, and $120 \mathrm{~min}$, respectively. Averaged over the entire exposure area and time, the total dose delivered was $7.19 \times 10^{14}$ electrons with a fluence of $2.05 \times 10^{6} \mathrm{~nm}^{-2}$ and flux of $285 \mathrm{~nm}^{-2} \mathrm{~s}^{-1}$.

After electron exposure, the droplet was evaporated by withdrawing the $\mathrm{NC}$ and pumping the specimen chamber to high vacuum. As illustrated in Fig. 2b, examination of the resulting deposit revealed a significant volume of $\mathrm{Cu}$ inside the large trenches that were milled back into the Si substrate, whereas the $\mathrm{SiO}_{2}$ surface contained only very small, sporadic $\mathrm{Cu}$ deposits. Fig. 3 is an electron micrograph of the electronirradiated region showing that the deeper Si trenches contained most of the electrodeposited $\mathrm{Cu}$, a result confirmed by energy dispersive X-ray spectroscopy (EDS) ${ }^{31}$ of the sample.

Due to the extremely low deposition rate observed on $\mathrm{SiO}_{2}$, a second BIVC experiment was performed using a much higher electron beam current density, and a region of $\mathrm{SiO}_{2}$ that did not contain any trenches. The current density was increased by over an order of magnitude by decreasing the exposure area from $\sim 350 \mu^{2}$ to $\sim 16 \mu^{2}$ (the beam current and energy were unchanged, and the exposure time was $30 \mathrm{~min}$ ). The total dose delivered was $1.8 \times 10^{14}$ electrons with a fluence of $1.12 \times$ $10^{7} \mathrm{~nm}^{-2}$ and flux of $6.24 \times 10^{3} \mathrm{~nm}^{-2} \mathrm{~s}^{-1}$. Fig. 4 is an SEM image of the resulting $\mathrm{Cu}$ deposit, which shows some degree of 


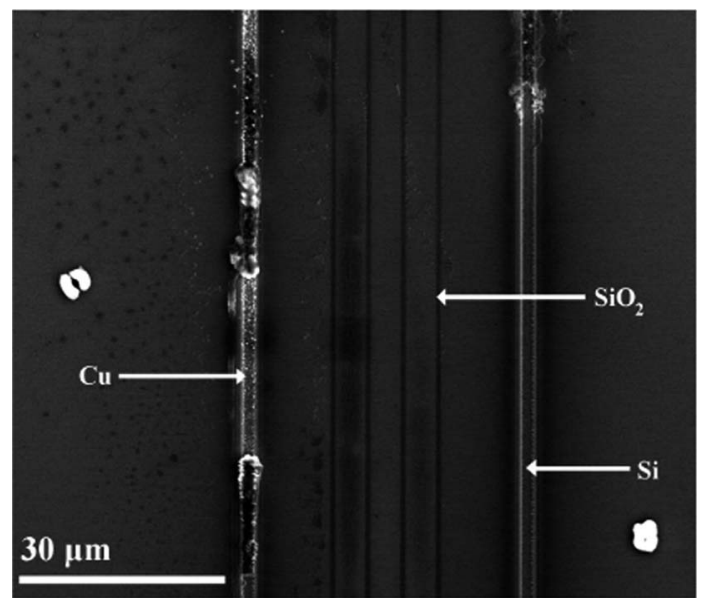

Fig. 3 Scanning electron micrograph of a BIVC experimental area taken after $\mathrm{Cu}$ deposition and subsequent dehydration of the sample. Both the image and EDS analysis show that electrodeposition of $\mathrm{Cu}$ occurred at much higher rates on $\mathrm{Si}$ than on $\mathrm{SiO}_{2}$.

localization to the exposure region. However, numerous $\mathrm{Cu}$ grains are randomly distributed around the region irradiated by the electron beam. Such grains were observed to nucleate at the edges of the liquid droplet and float along the droplet (likely influenced by local fluctuations in the electric field). When electron exposure was completed and the sample was dehydrated, these suspended $\mathrm{Cu}$ grains adhered to random locations on the $\mathrm{SiO}_{2}$ sample.

We note that the $\mathrm{Cu}$ deposition rate for comparable electron fluence on $\mathrm{SiO}_{2}$ was small relative to the that on $\mathrm{Si}$ (i.e., inside the trenches etched through $\mathrm{SiO}_{2}$ as shown in Fig. 2b). Clearly, the substrate has a dramatic impact on deposition rate, thus necessitating relatively high current density on insulating substrates as compared to conductive substrates. Hence, the BCVC experimental configuration was used to further test the role of substrate conductivity in deposition efficiency and the degree of deposit localization.

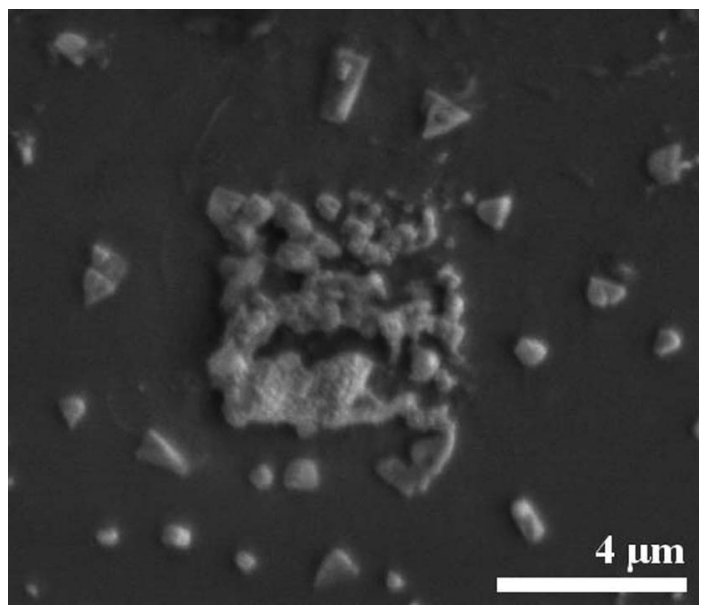

Fig. 4 Scanning electron micrograph of a BIVC experiment conducted on $\mathrm{SiO}_{2}$ The image was taken following Cu deposition and subsequent dehydration of the sample.
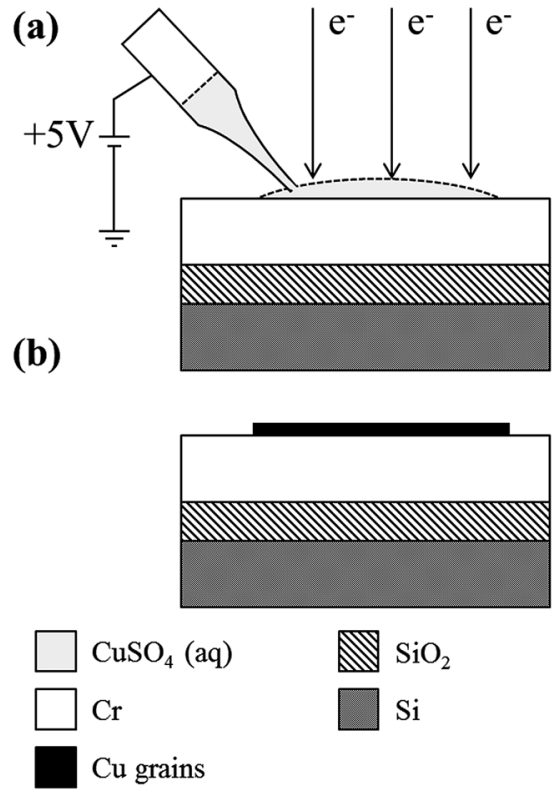

Fig. 5 Schematic illustration of the BCVC configuration used for electron beam induced electrodeposition of Cu (a). Copper deposition was localized to the area scanned by the electron beam (b).

The BCVC experimental configuration is illustrated in Fig. 5a. The sample used was a $500 \mathrm{~nm}$ film of $\mathrm{Cr}$ that had been sputtered onto a $1 \mu \mathrm{m}$ film of thermal $\mathrm{SiO}_{2}$ (on a Si substrate). $\mathrm{CuSO}_{4}$ was delivered to the $\mathrm{Cr}$ surface and the liquid was stabilized as discussed above. After flow was stabilized, the sample was lowered a few microns to ensure that the goldcoated NC was not contacting the $\mathrm{Cr}$ film as this would result in an electrical short of the galvanic circuit. The NC was then biased to $+5 \mathrm{~V}$ and a $5 \mathrm{keV}, 33 \mathrm{nA}$ electron beam was scanned for $30 \mathrm{~min}$ over a $600 \mu \mathrm{m}^{2}$ area of liquid-meniscus-covered $\mathrm{Cr}$, as shown in Fig. 5a. The total dose delivered was $3.7 \times 10^{14}$ electrons with a fluence of $6.18 \times 10^{5} \mathrm{~nm}^{-2}$ and flux of $343 \mathrm{~nm}^{-2}$ $\mathrm{s}^{-1}$. The formation of a thin meniscus, observed in this configuration, is attributed to the phenomenon of electrowetting $^{32}$ caused by the application of an electrical bias between the liquid and the metal surface.

The resulting $\mathrm{Cu}$ deposits were generally in the form of the exposure geometry, as shown in Fig. 5b. The deposition behavior was striking in that the rate was indeed much greater than that observed under similar electron beam exposure conditions used in the BIVC configuration. Fig. 6 is an SEM image of a $\mathrm{Cu}$ pad on the $\mathrm{Cr}$ film after dehydration in high vacuum. EDS analysis indicated that, as in the case of BIVC, the $\mathrm{Cu}$ deposited was of high purity. However, comparison of Fig. 4 and 6 exemplifies the vast difference in deposition rate. While the current density used in the BCVC configuration (Fig. 6, Cr substrate) was almost a factor of 40 lower (fluence nearly lower by a factor of two) than that used in the BIVC configuration (Fig. 4, $\mathrm{SiO}_{2}$ substrate), the deposited volume is much greater when the process is performed using a conductive substrate. A subsequent experiment in which a single exposure area overlapped both a $\mathrm{Cr}$ film and a region of $\mathrm{SiO}_{2}$ further confirmed the 


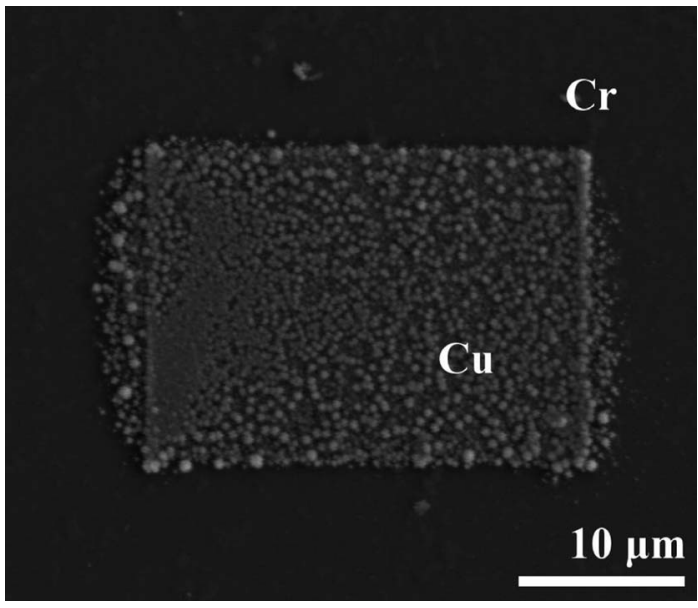

Fig. 6 Scanning electron micrograph of a BCVC experiment conducted on $\mathrm{Cr}$. The image was taken following Cu deposition and subsequent dehydration of the sample.

increase in deposition rate on the conductive film. In this case, the region of exposed Cr yielded a film similar to that seen in Fig. 6, while the exposed region of $\mathrm{SiO}_{2}$ showed negligible deposition.

In an effort to utilize the large difference between the BCVC and BIVC process rates, a methodology was devised in which IBID or EBID is used to fabricate seed layers that can mediate BCVC and localize electrodeposition on insulating substrates, as shown schematically in Fig. 7. Experimental validation of such a process was carried out using the $\mathrm{SiO}_{2}$-on-Si sample used in the BIVC configuration. Prior to liquid delivery, the system was pumped to high vacuum, and a set of Pt lines was deposited

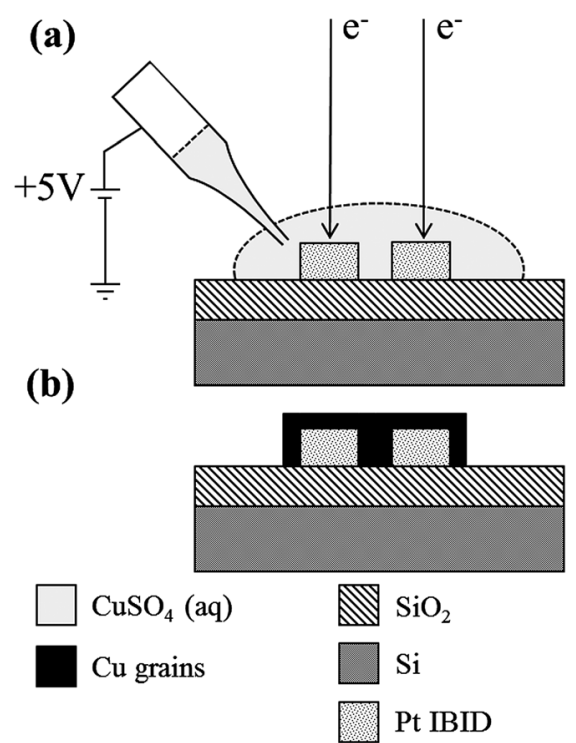

Fig. 7 Schematic illustration of the pre-patterned BCVC configuration used for electron beam induced electrodeposition. Metallic deposits pre-patterned in situ by Pt IBID on $\mathrm{SiO}_{2}$ serve as seed layers for electrodeposition of $\mathrm{Cu}(\mathrm{a})$. The final electrodeposit is spatially localized by Pt IBID (b), and the electrodeposition rate exceeds that of the BIVC process on $\mathrm{SiO}_{2}$. by IBID using a $1 \mathrm{nA}$ ion beam and trimethyl(methylcyclopentadienyl)Pt vapor as the growth precursor (i.e., a common precursor $^{15}$ for EBID and IBID of Pt).

Following IBID, the vacuum system was switched to ESEM mode and an electrolyte droplet was stabilized on the surface. As before, the sample was lowered a few microns to ensure there was no direct electrical contact between the NC and the Pt. A $30 \mathrm{keV}, 16 \mathrm{nA}$ electron beam was used to irradiate an area of $\sim 200 \mu \mathrm{m}^{2}$ so as to achieve a spatially averaged current density similar to that used in the above BCVC configuration. The exposure was carried out for 60 minutes resulting in a total dose of $3.6 \times 10^{14}$ electrons with a fluence of $1.8 \times 10^{6} \mathrm{~nm}^{-2}$ and flux of $500 \mathrm{~nm}^{-2} \mathrm{~s}^{-1}$ averaged over the entire exposure region. The irradiated area overlapped two $\mathrm{Pt}$ lines and the $\mathrm{SiO}_{2}$ substrate (Fig. 7a).

After $\mathrm{Cu}$ electrodeposition was completed, the sample was dehydrated by pumping the system to high vacuum. Fig. $8 \mathrm{a}$ is an SEM image of the processed substrate. As expected, $\mathrm{Cu}$ deposition was initiated and occurred primarily on and around the
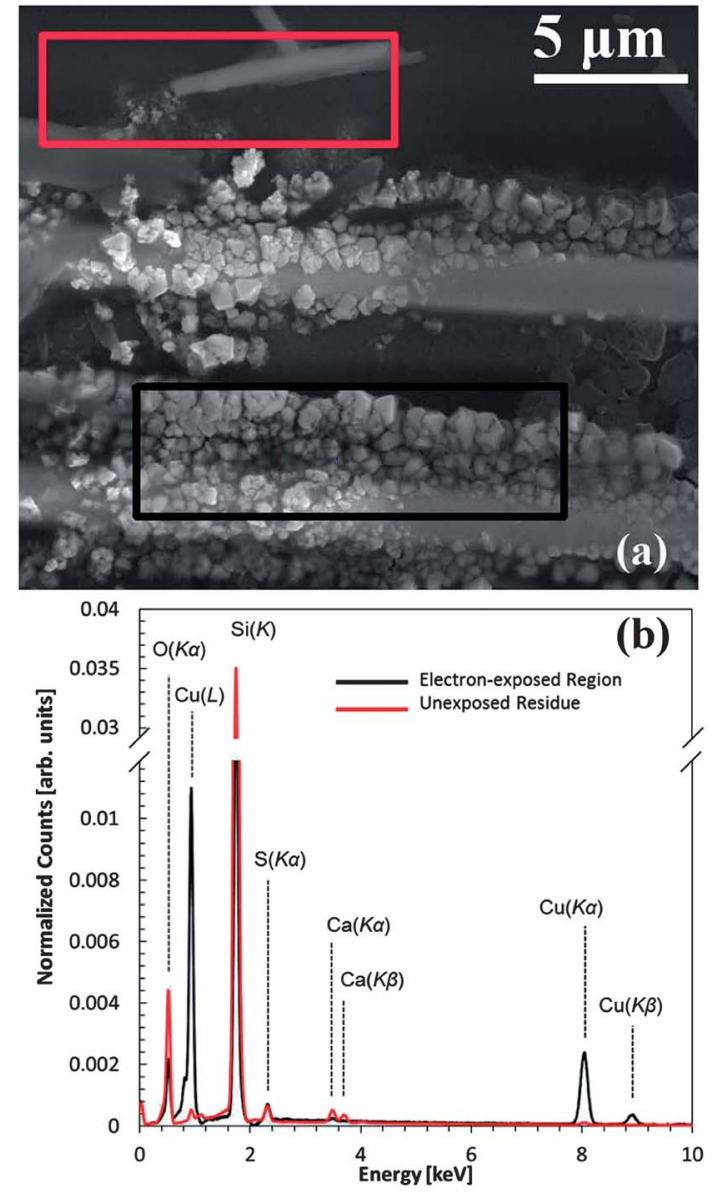

Fig. 8 Scanning electron micrograph (a) and selected area EDS scans (b) of an IBID-seeded BCVC experiment conducted on $\mathrm{SiO}_{2}$. The images were taken following $\mathrm{Cu}$ deposition and subsequent dehydration of the sample. The rectangular region in enclosed in red was not exposed to electrons and is comprised primarily of residue from dehydration of the solution. The region enclosed in black contains a localized Cu deposit in close proximity to a Pt deposit. EDS scans in (b) show a much higher $\mathrm{Cu}: \mathrm{S}$ ratio in the region near the Pt feature. 
IBID-grown Pt. EDS spectra confirming this to be the case are shown in Fig. 8b. The two spectra in Fig. $8 \mathrm{~b}$ correspond to the two regions in Fig. 8a where the red and black rectangles enclose regions without and with electron exposure, respectively. The elemental analysis of the unexposed region reveals traces of $\mathrm{Ca}, \mathrm{Cu}, \mathrm{S}$, and $\mathrm{O}$ (in addition to $\mathrm{Si}$ and $\mathrm{O}$ from the underlying substrate), which correspond to salt residues such as $\mathrm{CaSO}_{4}$ (a side reaction with trace $\mathrm{Ca}$ in water) and $\mathrm{CuSO}_{4}$. When the Pt background is subtracted and the spectra are normalized to $\mathrm{S}$, it becomes clear that locally, there is a higher quantity of $\mathrm{Cu}$ in the regions near the IBID-grown Pt deposit. Electrodeposition appears to have propagated from the Pt lines, partially filling the space between them.

While this experiment showed the feasibility of using IBID seed layers to localize electron beam induced electrodeposition on an insulating substrate, further work remains to improve control of the technique. In particular, the continuity of the $\mathrm{Cu}$ deposit seen in Fig. 8 is poor, and the EDS spectra in Fig. $8 \mathrm{~b}$ reveal the presence of trace contaminants in the form of $\mathrm{Ca}$ and $\mathrm{Cu}$ salt residue on the dehydrated substrate. Some of the salt residue problems following dehydration may be minimized by using highly diluted precursor solutions, but this will negatively impact the rate of the process. Likely post-processing will be necessary (preferably in situ) to eliminate the residual salts. Regardless, mitigating or removing salt contaminants from the samples must be addressed in order to improve the process.

\section{Discussion}

For the process of electrochemical deposition to occur, in general, there are several basic requisites that should be met. Among these are: (1) electrolyte should wet the substrate; (2) solvated cations should be transported to the interface; (3) solvated cations become dehydrated due to electric fields present at the interface; (4) charge is transferred resulting in reduction of the cation; (5) the charge transfer should be balanced to maintain charge neutrality of the system. To highlight the mechanisms that likely underpin the behavior reported here, we will discuss the results in the context of these requisites.

In the following discussion, we assume that the beam electrons can traverse the droplet and penetrate the electrolytesubstrate interface. This assumption is justified because electrodeposition was localized to the substrate region scanned by the beam (see Fig. 4 and 6). Furthermore, it is consistent with Monte Carlo simulations of electron range in water. ${ }^{33,34}$

\subsection{Wetting}

Wetting of a substrate is necessary as ion transport from the liquid to the surface requires contact between the two phases at the interface. The wettability of a solid surface is largely governed by thermodynamic forces, but the contact angle (i.e., wetting ability), $\theta$, of a liquid-solid interface can be modified by means of an applied voltage through the phenomenon of electrowetting. ${ }^{35}$ Given the experimental setup used in this work, electrowetting may play a key role in beam induced electrochemistry.
For cases such as the BCVC method presented here, where deposition is performed on an electrical conductor, the voltage required to significantly alter wetting is on the order of $10^{2} \mathrm{mV} .^{35}$ Conversely, in the case of BIVC, the voltage required to drive wetting of the insulating substrate is significantly higher, ${ }^{35}$ and may contribute to the discrepancy in deposition rates between the BIVC and BCVC processes. Specifically, electrowetting is expected to play a significant role only in the case of BCVC, yielding high surface coverage of the electrolyte and an efficient supply rate of $\mathrm{Cu}^{2+}$ to the solid-liquid interface. Conversely, inefficient wetting of insulating substrates likely contributes to the low deposition rate on $\mathrm{SiO}_{2}$ relative to the high rates observed on conductive substrates (e.g. Si, Cr, or Pt IBID).

In BIVC experiments, electrowetting may be altered at the micro scale by sub-surface charging of the substrate. ${ }^{36-38}$ Such charging is caused by the electron beam, and occurs within the electron interaction volume in the insulator. The corresponding electric fields are highly non-uniform, fluctuate as the beam is scanned over a substrate, and are expected to alter electrowetting and contribute to the sporadicity of deposition observed on insulating substrates.

\subsection{Charge transport}

The above discussion was focused on electrolyte wetting which affects cation transport to the liquid-solid interface. However, it is possible that electron transfer and supply rates limit the rate of the BIVC process. When the electron beam impinges on an electrolyte-substrate interface, it excites electrons throughout the interaction volume and injects excess electrons into the substrate..$^{37}$ These electrons thermalize ${ }^{39}$ and likely play a role in the electrodeposition process where their supply rate is influenced by transport through the substrate. In the case of an insulator, transport is inefficient and driven primarily by the non-uniform, sub-surface electric field generated as a result of charging. ${ }^{37}$ Consequently, the electron supply rate likely plays a significant role in the slow and sporadic nature of the deposition process observed on insulators.

The discussion of electron transfer brings to light a key difference between how electrons are supplied to the electrolyte-solid interface compared to standard electrochemical methods. Rather than being sourced from a conductive cathode, the situation here involves direct injection and excitation of electrons by the beam. In standard electrochemistry, the structure of the metal-electrolyte interface has been studied extensively and is well understood ${ }^{40}$ in terms of a Galvani potential and an electric double layer (EDL) comprised of a bilayer of opposite charge accumulated at the interface. The small (molecular) thickness of the EDL is responsible for the extraordinarily high capacitance encountered at the interface as discussed previously in the context of electrowetting. The electrostatic nature of the EDL implies that significant disruption of the charge distribution is expected in the case of electron bombardment. It would be highly speculative to address the nature of this disruption, but the likelihood of its occurrence warrants inclusion in the present discussion. 
Finally, considering the role of the electron beam, a brief discussion of solvated electrons and solvated cations is warranted. Solvated electrons have been observed and studied in many electrochemical systems. ${ }^{41}$ More recently, carbon nanostructures have been demonstrated as a means of cathodicallygenerating solvated electrons $\mathbf{4}^{\mathbf{4 2}}$ for electrochemistry. In electron beam induced electrochemistry, it is possible that the beam acts as a source of solvated electrons and thus plays a direct, active role in the electrochemical system by means of electron injection and excitation throughout the interaction volume. While clearly speculative at present, the generation of solvated electrons should be considered in future work on the theory of beam induced electrochemistry.

\section{Conclusion}

Localized delivery of liquids onto solid substrates inside the vacuum chamber of an electron microscope was demonstrated using borosilicate nanocapillaries. Droplets were stabilized by balancing capillary flow with the droplet evaporation rate. The technique has enabled electron beam induced electrodeposition of $\mathrm{Cu}$, achieved by delivering $\mathrm{CuSO}_{4}$ (aq.) into an environmental scanning electron microscope, and by irradiating electrolyte droplets with an electron beam. Deposition onto electrical conductors and insulators was demonstrated and mechanistic differences between the two systems were discussed. Electrodeposition on insulators is characterized by slow, intermittent deposition attributed to a number of mechanisms that likely include poor wetting and charging of the substrate. Conversely, conductive substrates were observed to mediate rapid, localized electrodeposition. Deterministic electrodeposition onto insulators was achieved using Pt seed layers that had been pre-fabricated by ion beam induced deposition. Further work is needed to improve the uniformity of the electrodeposited $\mathrm{Cu}$ and to eliminate residual salt contaminants observed to precipitate on the substrate. The liquid delivery technique opens new avenues for interfacing microfluidic systems to a vacuum chamber, and electron beam studies of liquid-solid reactions, wetting and spreading behavior at the micro and nano scales.

\section{References}

1 D. F. Parsons, Science, 1974, 186, 407.

2 N. de Jonge and F. M. Ross, Nat. Nanotechnol., 2011, 6, 695704.

3 E. U. Donev and J. T. Hastings, Nano Lett., 2009, 9, 27152718.

4 E. U. Donev and J. T. Hastings, Nanotechnology, 2009, 20, 505302.

5 G. Schardein, E. U. Donev and J. T. Hastings, Nanotechnology, 2011, 22, 015301.

6 E. U. Donev, G. Schardein, J. C. Wright and J. T. Hastings, Nanoscale, 2011, 3, 2709-2717.

7 Y. Liu, X. Chen, K. W. Noh and S. J. Dillon, Nanotechnology, 2012, 23, 385302.
8 K. Rykaczewski and J. H. J. Scott, ACS Nano, 2011, 5, 59625968.

9 K. Rykaczewski, J. H. J. Scott and A. G. Fedorov, Appl. Phys. Lett., 2011, 98, 093106.

10 K. Rykaczewski, T. Landin, M. L. Walker, J. H. J. Scott and K. K. Varanasi, ACS Nano, 2012, 6, 9326-9334.

11 N. A. Stelmashenko, J. P. Craven, A. M. Donald, E. M. Terentjev and B. L. Thiel, J. Microsc., 2001, 204, 172183.

12 D. J. Stokes, B. L. Thiel and A. M. Donald, Langmuir, 1998, 14, 4402-4408.

13 D. P. DePonte, R. B. Doak, M. Hunter, Z. Liu, U. Weierstall and J. C. H. Spence, Micron, 2009, 40, 507-509.

14 D. P. Deponte, J. T. McKeown, U. Weierstall, R. B. Doak and J. C. H. Spence, Ultramicroscopy, 2011, 111, 824-827.

15 I. Utke, S. Moshkalev and P. Russell, Nanofabrication Using Focused Ion and Electron Beams: Principles and Applications, Oxford University Press, New York, 2012.

16 S. J. Randolph, J. D. Fowlkes and P. D. Rack, Crit. Rev. Solid State Mater. Sci., 2006, 31, 55-89.

17 I. Utke, P. Hoffmann and J. Melngailis, J. Vac. Sci. Technol., B: Microelectron. Nanometer Struct.-Process., Meas., Phenom., 2008, 26, 1197-1276.

18 W. F. Van Dorp and C. W. Hagen, J. Appl. Phys., 2008, 104, 081301.

19 J. D. Wnuk, J. M. Gorham, S. G. Rosenberg, W. F. Van Dorp, T. E. Madey, C. W. Hagen and D. H. Fairbrother, J. Phys. Chem. C, 2009, 113, 2487-2496.

20 J. D. Wnuk, S. G. Rosenberg, J. M. Gorham, W. F. Van Dorp, C. W. Hagen and D. H. Fairbrother, Surf. Sci., 2011, 605, 257266.

21 K. Landheer, S. G. Rosenberg, L. Bernau, P. Swiderek, I. Utke, C. W. Hagen and D. H. Fairbrother, J. Phys. Chem. C, 2011, 115, 17452-17463.

22 A. Botman, J. J. L. Mulders and C. W. Hagen, Nanotechnology, 2009, 20, 372001.

23 J. J. L. Mulders, L. M. Belova and A. Riazanova, Nanotechnology, 2011, 22, 055302.

24 J. Bishop, C. J. Lobo, A. Martin, M. Ford, M. Phillips and M. Toth, Phys. Rev. Lett., 2012, 109, 146103.

25 A. Botman, M. Hesselberth and J. J. L. Mulders, J. Vac. Sci. Technol., B: Microelectron. Nanometer Struct.-Process., Meas., Phenom., 2008, 26, 2464-2467.

26 K. L. Klein, S. J. Randolph, J. D. Fowlkes, L. F. Allard, H. M. Meyer III, M. L. Simpson and P. D. Rack, Nanotechnology, 2008, 19, 345705.

27 G. D. Danilatos, Adv. Electron. Electron Phys., 1988, 71, 109250.

28 A. M. Donald, Nat. Mater., 2003, 2, 511-516.

29 B. L. Thiel and M. Toth, J. Appl. Phys., 2005, 97, 051101.

30 Focused Ion Beam Systems: Basics and Applications, ed. N. Yao, Cambridge University Press, Cambridge, 2011.

31 D. E. Newbury, D. C. Joy, P. Echlin, C. Fiori and J. Goldstein, Advanced Scanning Electron Microscopy and X-Ray Microanalysis, Springer, 1986.

32 T. Squires and S. Quake, Rev. Mod. Phys., 2005, 77, 9781016. 
33 K. Wiklund, J. M. Fernandez-Varea and B. K. Lind, Phys. Med. Biol., 2011, 56, 1985-2003.

34 J. Ding-Ju and T. Zhen-Yu, Chin. Phys. Lett., 2010, 27, 033401. 35 C. Quilliet and B. Berge, Curr. Opin. Colloid Interface Sci., 2001, 6, 34-39.

36 M. Toth, S. O. Kucheyev, J. S. Williams, C. Jagadish, M. R. Phillips and G. Li, Appl. Phys. Lett., 2000, 77, 13421344.

37 M. Toth, W. R. Knowles and M. R. Phillips, Appl. Phys. Lett., 2007, 90, 072905.
38 M. Toth, W. R. Knowles and B. L. Thiel, Appl. Phys. Lett., 2006, 88, 023105.

39 H. J. Fitting, E. Schreiber, Kuhr and A. von Czarnowski, J. Electron Spectrosc. Relat. Phenom., 2001, 119, 35-47.

40 V. Bagotsky, Fundamentals of Electrochemistry, John Wiley and Sons Inc., Hoboken, NJ, 2nd edn, 2006, p. 722.

41 L. I. Krishtalik and N. M. Alpatova, Sov. Electrochem., 1976, 12, 161-190.

42 A. G. Krivenko, N. S. Komarova and N. P. Piven', Electrochem. Commun., 2007, 9, 2364-2369. 